\title{
Motion Law Analysis and Structural Optimization of the Ejection Device of Tray Seeder
}

\author{
Xin $\mathrm{Luo}^{1, *}, \mathrm{Bin} \mathrm{Hu}^{1}$, Chunwang Dong ${ }^{1,2}$, and Lili Huang ${ }^{1}$ \\ ${ }^{1}$ Machinery and Electricity Engineering College, Shihezi University, Shihezi 832003,China \\ Tel.:13031336692 \\ ${ }^{2}$ Insititute of Cotton; The Chinese Academy of Agricultural Sciences; Anyang; \\ Henan 455000 China \\ hb_mac@shzu.edu.cn
}

\begin{abstract}
An ejection mechanism consisting four reset springs, an electromagnet and a seed disk was designed for tray seeder. The motion conditions of seeds in the seed disk were theoretical analyzed and intensity and height of seed ejection were calculated. The motions of the seeds and seed disk were multi-body dynamic simulated using Cosmos modules plug-in SolidWorks software package. The simulation results showed the consistence with the theoretical analysis.
\end{abstract}

Keywords: Tray seeders; ejection mechanism; numerical simulation; motion analysis.

\section{Introduction}

The Tray seeder is an essential component for modern cultivation techniques. Whether seeds are vibrated uniformly to suspension state by vibration device can affect the seeding capability. Now continual excited vibration by electromagnetic vibration device has been extensively employed to make the seeds "bouncing" and enable them being sucked. However, the uneven vibration intensity of seeds in different positions causes the seeds suspend in different height, so seeder has the poor air-suction effect for seeds. In recent years, the motion law of seeds based on continual excited vibration has been studied (cheng jin, 2002; Li Yaoming et al, 2009; Zhao Lixin et al, 2003). They reported that the seeds should obtain enough inertia force to get in appropriate suspension station for satisfaction of the air-suction requirements. But there is lack of information on how to make all seeds in different positions acquire same vibrating intensity. In this paper, an ejection mechanism consisting four reset springs, a electromagnet and a seed disk was designed. The motion conditions of seeders in the seed disk were theoretical analyzed and then ejection intensity and height were calculated. The motions of the seeds and seed disk were multi-body dynamic simulated using Cosmos modules plug-in SolidWorks software package. The simulation results showed the consistence with the theoretical analysis.

* Corresponding author. 


\section{Working Principle of Ejection Mechanism}

The ejection mechanism schematic diagram is shown in Fig.1. It mainly consists of four reset springs, a electromagnet and a seed disk. The disk and the armature of electromagnet are connected together by pin bolts. The reset springs are installed in the lug boss between the worktable and the seed disk. The armature overcomes friction, pulls the disk down when the electromagnet is charged, and conversely, the disk is ejected by the reset springs and the seeds are tossed into suspension. The hopping seeds can overcome the force among seeds, effectively improving the adsorption rate of all seeds.

Structural Design Parameters :

The weight of disk is $3 \mathrm{~kg}$, the stiffness of spring is $4 \mathrm{~N} / \mathrm{mm}$, the voltage of electromagnet is $220 \mathrm{v}$, the maximum traction force is $80 \mathrm{~N}$, and the maximum stroke is $25 \mathrm{~mm}$.

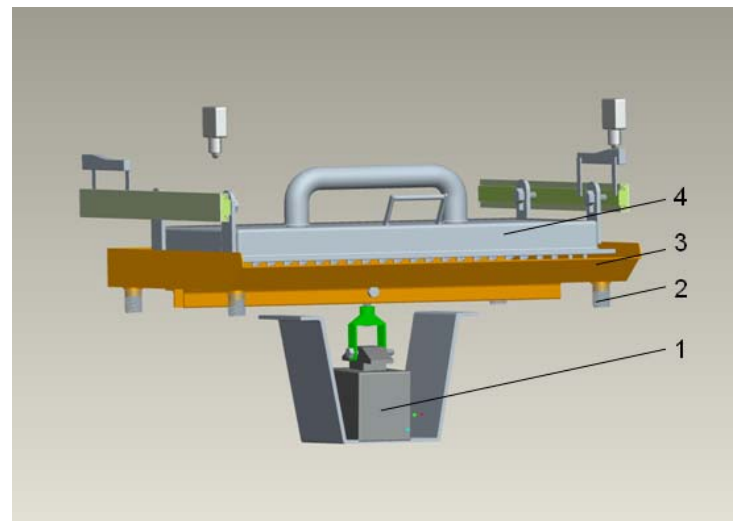

1. electromagnet 2. reset spring 3.disk 4.sucker

Fig. 1. schematic diagram of ejection mechanism

\section{Analysis of Motion Regularity of Ejection Mechanism}

\subsection{Moving Condition and Calculation for Hopping of Seeds}

The movements of monolayer seeds on the disk are studied. Gravity and force of inertia of the seeds are mainly considered (J. Theuerkauf P. Witt, 2006), air resistance is neglected. The critical condition for hopping of seeds is:

$$
\mathrm{P} \geq \mathrm{G}
$$

Where: $P$ is the inertia force of seeds $(\mathrm{N}), \mathrm{G}$ is weight of seeds $(\mathrm{N})$

The periodical harmonic excitation equation of disk is:

$$
x=A \mathrm{e}^{-\varepsilon t} \sin \left(\omega_{d} t+\varphi\right)
$$


Without considering the system damping, the motion is:

$$
x=A \sin (\omega t+\varphi)
$$

Where: $\mathrm{t}$ is vibration time(s), $\varphi$ is the initial phase of vibration(rad), $\varphi$ is natural frequency $(\mathrm{Hz})$.

The initial acceleration of seeds is equal to disk's:

$$
\alpha_{s}=\alpha_{d}=A \omega^{2}
$$

According to Newton Second Law:

$$
P=m \alpha_{s}=\frac{G}{g} A \omega^{2}
$$

Puts (5) into (1):

$$
P=\frac{G}{g} A \omega^{2} \geq G
$$

The ejection strength coefficient $K$ is:

$$
K=\frac{A \omega^{2}}{g} \geq 1
$$

When $\mathrm{k}$ is greater than 1 , seeds are tossed.

As shows in Fig.2,

$$
\begin{gathered}
P+(M+m) g=k(\Delta x+\delta) \\
\delta=\frac{(M+m) g}{k}
\end{gathered}
$$

Where: $k$ is the coefficient of stiffness of spring $\Delta x$ is the amount of compression, $\delta$ is the static Deformation between seed and disk.

Puts (9) into (8):

$$
k=P / \Delta x
$$

According to the structural characteristics of the ejection mechanism:

$\mathrm{P}=80 \mathrm{~N}, \Delta x=20 \mathrm{~mm}, k=4 \mathrm{~N} / \mathrm{mm}$.

The natural frequency of the ejection system is:

$$
\omega=\sqrt{\frac{\kappa}{m_{s}}}=\sqrt{\frac{4 \times 1000}{3}}=36.5 \mathrm{rad} / \mathrm{s}
$$

Where: $m_{s}$ is the total mass of seed and disk 


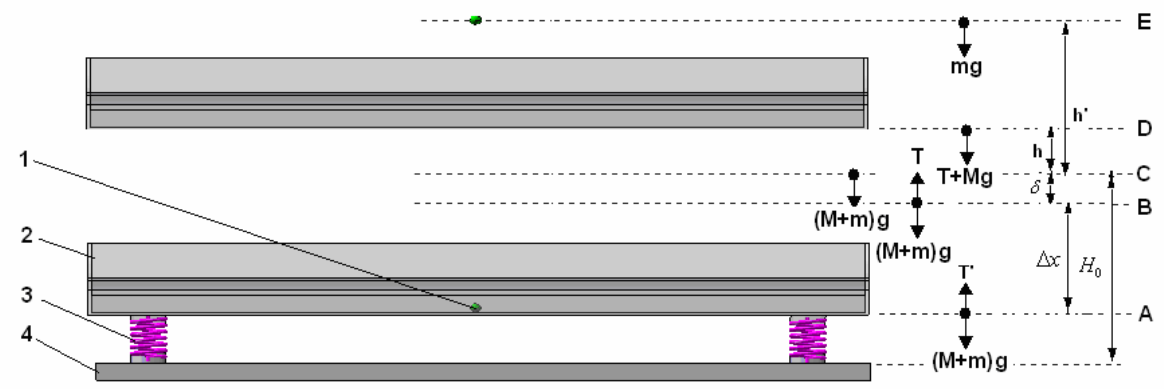

1. cotton seed 2. disk 3. reset spring

Fig. 2. Motion State of ejection mechanism

The amplitudes of disk are:

$$
A=\sqrt{x_{t 1}{ }^{2}+\left(\frac{x_{t 1}}{\omega_{n}}\right)^{2}}=\sqrt{20^{2}+0}=20 \mathrm{~mm}
$$

Put it into (7)

$$
K=\frac{A \omega^{2}}{g}=\frac{0.02 \times 36.5^{2}}{9.8}=2.718 \geq 1
$$

The results meet the requirement.

\subsection{Analysis on Ejecting Height of Seed}

As shown in Fig.2, at the position of B, According to the conservation of mechanical energy:

$$
\frac{1}{2}(M+m) v^{2}+(M+m) g \Delta x+\frac{1}{2} k \delta^{2}=E s
$$

Elastic potential energy is

$$
E s=\frac{1}{2} k(\Delta x+\delta)^{2}
$$

Puts (12) into (11)

$$
v=\sqrt{\frac{k \Delta x^{2}(\Delta x+\delta)}{(M+m)}-2 g \Delta x}
$$

Where $\mathrm{M}$ is the weight of the disk, $m$ is the weight of seeds, $v$ is the speed before separation.

Throwing height of seed is:

$$
h^{\prime}=\frac{v^{2}}{2 g}=\frac{k \Delta x^{2}(\Delta x+\delta)}{2 g(M+m)}-\Delta x
$$


According to the conservation of mechanical energy:

$$
\begin{gathered}
k h^{2}+2 M g h-M v^{2}+2 M g \delta-k \delta^{2}=0 \\
h=\sqrt{\frac{M v^{2}+k \delta^{2}}{k}-\frac{2 M g \delta}{k}}-\frac{M g}{k}
\end{gathered}
$$

Puts $\mathrm{M}=33 \mathrm{~kg}, \mathrm{~m}=0.096 \mathrm{~g} \mathrm{P}=80 \mathrm{~N}$ into (11)、(13)、 (14)

$\mathrm{v}=735 \mathrm{~mm} / \mathrm{s}, \mathrm{h}=12.59 \mathrm{~mm}, \mathrm{~h}^{\prime}=26.67 \mathrm{~mm}, \mathrm{~h}^{\prime}-\mathrm{h}=14.08 \mathrm{~mm}$.

\section{Multi-Body Dynamics Simulation of the Ejection Vibration Mechanism}

\subsection{Model Establishment of Ejection Mechanism}

The ejection mechanism model consisting of seed disk, seeds and working table was established using SolidWorks software. In the seamless simulation environment of CosmosMotion, the distance between seed disk and working table was set $42.5 \mathrm{~mm}$, namely, electromagnet can draw spring $27.5 \mathrm{~mm}$ from original position.

\subsection{Definition of Constraint}

The constraints of parts in ejection mechanism model can be automatically created in CosmosMotion environment according the mating counterpart. Seed disk and seeds were defined as motion body and working table was defined as stationary body. The simulation parameters were set as follows: material of disk is alloy steel; density of cotton seed is $1.06 \times 103 \mathrm{~kg} / \mathrm{m} 3$, weight is $0.96 \mathrm{~g}$, restitution coefficient is 0.5 , friction coefficient is 0.3 ; Stiffness of each reset spring (adding four spring between seed disk and spring seat) is $1 \mathrm{~N} / \mathrm{mm}$, length is $70 \mathrm{~mm}$, diameter is $16 \mathrm{~mm}$, wire diameter is $1.6 \mathrm{~mm}$, cycle number is $11.3 \mathrm{~d}$. $3 \mathrm{~d}$ elastic collision between disk and seeds is defined (friction is set dynamic).The parameters of equation solver was defined as follows: ADAMS GSTIFF was set $0.5 \mathrm{~s}$; frame numbers was 100 and other parameters are defaulted.

\subsection{Simulation Results}

Mesh was automatically calculated by equation solver. The curve of velocity and centroid displacement of seed and disk are as shown in fig 4 (red represents seeds, blue represents disk). The Seed moved to a static balance position while the disk reached the maximal rate, and then they began to separate. Subsequently the seed was ejected and the disk started to decrease in velocity, which is consistent with the theoretical analysis. The highest ejection height of seed above disk was $13.5 \mathrm{~mm}$, which is close to the theoretical calculated value of $14.08 \mathrm{~mm}$. The difference between values comes from the energy loss caused by collision friction. 


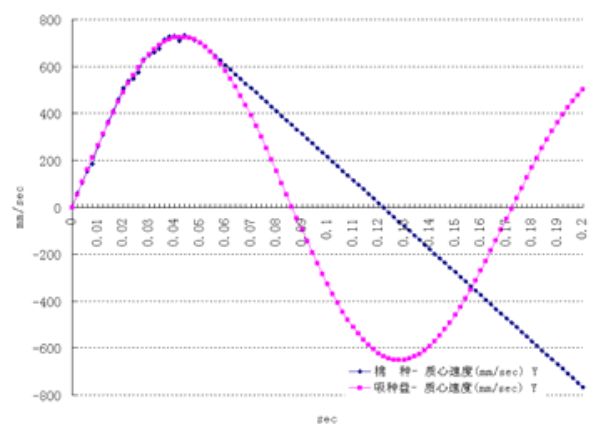

Fig. 3. Motion trajectory of speed

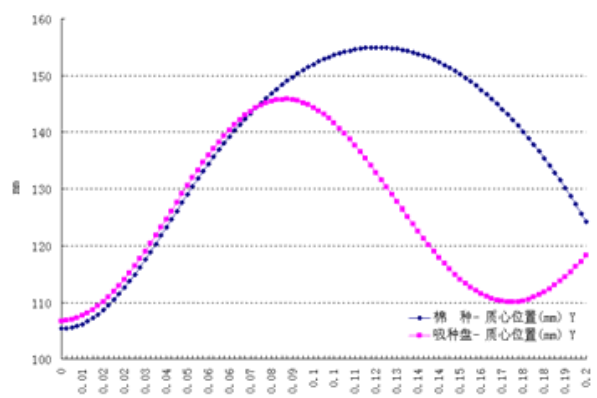

Fig. 4. Motion trajectory of centroid position

\section{Conclusion}

1. The results of theoretical analysis on ejection mechanism motion showed that seed could be ejected completely when the coefficient of stiffness of spring $k \geq 1$. The ejection height was determined by the static deformation between seed and disk $(\delta)$, ejection velocity $(v)$, the coefficient of stiffness of spring $(k)$.

2. Motion simulation of disk and seed showed the consistence with the results of theoretical analysis. The difference between values comes from the energy loss caused by collision friction. When the seed moved to the static balance position together with the seed disk, they began to separate for their velocities decrease at different rate.

3. The physical prototype is simple, which has a reliable performance and the rising height of the seeds are even. The tests have confirmed that the ratio of single-seed was over $97 \%$, The ratio of non-seed was below $2 \%$, completely to satisfy the requirements of precision seeder. 


\section{References}

[1] Jin, C., Yaoming, L.: Study on Seeds Movement Law in Sowing Test Stand with Suction and Vibration. Transactions of The Chinese Society of Agricultural Machinery 33(1), 47-50 (2002)

[2] Yaoming, L., Zhan, Z., Jin, C., Lizhang, X.: Discrete Element Method Simulation of Seeds Motion in Vibrated Bed of Precision Vacuum Seeder. Transactions of The Chinese Society of Agricultural Machinery (03), 55-59 (2009)

[3] Yaoming, L., Zhan, Z., Jin, C., Lizhang, X.: Numerical Simulation and Experiment on the Seeds Pickup Performance of Precision Air-suction Seeder. Transactions of The Chinese Society of Agricultural Machinery 39(10), 95-100 (2008)

[4] Lixin, Z., Liyun, Z.: Seed suction performance of vibrational air-suction tray seeder. Transactions of the Chinese Society of Agricultural Engineering 19(04), 122-125 (2003)

[5] Singh, R.C., Singh, G., Saraswat, D.C.: Optimisation of Design and Operational Parameters of a Pneumatic Seed Metering Device for Planting Cottonseeds. Biosystems Engineering 92(4), 429-438 (2005)

[6] Theuerkauf, J., Witt, P., Schwesig, D.: Analysis of particle porosity distribution in fixed beds using the discrete element method. Powder Technology 165(2), 92-99 (2006)

[7] Shangping, L., Yanmei, M., Fanglen, M.: Research on the working mechanism and virtual design for a brush shape cleaning element of a sugarcane harvester. Journal of Materials Processing Technology 129(1), 418-422 (2002)

[8] Bangchun, W.: Vibration Utilization Engineering, pp. 53-54. Science Press, Beijing (2005)

[9] Edited by SolidWorks Corporation. COSMOS Advanced Course: CosmosMotion. Machinery Industry publishing house, Beijing (July 2008)

[10] Kurowski, P.M.: Ph.D, P.Eng. Engineering Analysis with Cosmos Works Professional (2006) 